\title{
Centromere-targeted de novo integrations of an LTR retrotransposon of Arabidopsis lyrata
}

\author{
Sayuri Tsukahara, ${ }^{1,2,6}$ Akira Kawabe, ${ }^{3,6,7}$ Akie Kobayashi, ${ }^{1}$ Tasuku Ito, ${ }^{1,4}$ Tomoyuki Aizu, ${ }^{5}$ \\ Tadasu Shin-i, ${ }^{5}$ Atsushi Toyoda, ${ }^{5}$ Asao Fujiyama, ${ }^{5}$ Yoshiaki Tarutani, ${ }^{1,2}$ and Tetsuji Kakutani ${ }^{1,2,4,8}$ \\ ${ }^{1}$ Department of Integrated Genetics, National Institute of Genetics, Shizuoka 411-8540, Japan; ${ }^{2}$ Department of Genetics, School \\ of Life Science, The Graduate University for Advanced Studies (SOKENDAI), Shizuoka 411-8540, Japan; ${ }^{3}$ Department of \\ Population Genetics, National Institute of Genetics, Shizuoka 411-8540, Japan; ${ }^{4}$ Department of Biological Sciences, Graduate \\ School of Science, The University of Tokyo, Hongo, Bunkyo-ku, Tokyo 113-0033, Japan; ${ }^{5}$ Center for Genetic Resource \\ Information, National Institute of Genetics, Shizuoka 411-8540, Japan
}

\begin{abstract}
The plant genome evolves with rapid proliferation of LTR-type retrotransposons, which is associated with their clustered accumulation in gene-poor regions, such as centromeres. Despite their major role for plant genome evolution, no mobile LTR element with targeted integration into gene-poor regions has been identified in plants. Here, we report such targeted integrations de novo. We and others have previously shown that an ATCOPIA93 family retrotransposon in Arabidopsis thaliana is mobilized when the DNA methylation machinery is compromised. Although ATCOPIA93 family elements are low copy number in the wild-type $A$. thaliana genome, high-copynumber related elements are found in the wild-type Arabidopsis lyrata genome, and they show centromere-specific localization. To understand the mechanisms for the clustered accumulation of the A. lyrata elements directly, we introduced one of them, named Tal1 (Transposon of Arabidopsis lyrata 1), into A. thaliana by transformation. The introduced Tal1 was retrotransposed in A. thaliana, and most of the retrotransposed copies were found in centromeric repeats of $A$. thaliana, suggesting targeted integration. The targeted integration is especially surprising because the centromeric repeat sequences differ considerably between $A$. lyrata and $A$. thaliana. Our results revealed unexpectedly dynamic controls for evolution of the transposon-rich heterochromatic regions.
\end{abstract}

[Keywords: centromere; tandem repeat; retrotransposon; evolution]

Supplemental material is available for this article.

Received November 22, 2011; revised version accepted February 21, 2012.

Retrotransposons are major factors causing rapid evolution of plant genomes; comparison of genome structures in closely related plant species has revealed that the changes in plant genome size and organization are mainly caused by rapid proliferation and deletion of LTR retrotransposons (Hawkins et al. 2006; Vitte and Bennetzen 2006; Hu et al. 2011). Rapid proliferation of transposons would be a potential threat to the function of the host genome because each integration may directly disrupt a protein-coding region or indirectly perturb transcription of nearby genes. However, most of the LTR retrotransposons in plant genomes are accumulated in gene-poor

\footnotetext{
${ }^{6}$ These authors contributed equally to this work.

${ }^{7}$ Present address: Department of Bioresource and Environmental Sciences, Faculty of Life Sciences, Kyoto Sangyo University, Motoyama Kamigamo, Kyoto 606-8555, Japan.

${ }^{8}$ Corresponding author.

E-mail tkakutan@lab.nig.ac.jp.

Article published online ahead of print. Article and publication date are online at http://www.genesdev.org/cgi/doi/10.1101/gad.183871.111.
}

regions, resulting in proliferation of these elements with less harmful effects to the host (SanMiguel et al. 1996, 1998; Rabinowicz et al. 1999). This feature causes a differentiation of gene-rich regions and transposon-rich regions within plant genomes. The latter constitute heterochromatic domains, which play a significant role in large-scale chromosome organization and its behavior (Dawe and Hiatt 2004; Grewal and Jia 2007).

How are the biased distributions of LTR retrotransposons generated? One possibility is through natural selection; if chromosomal domains with deleterious transposon insertions into genic regions are eliminated from natural populations, the transposons would be condensed in genepoor regions. An alternative mechanism for the biased distribution is targeted integration. Targeted integrations of retrotransposons and retroviruses are known in yeast and mammals (Bushman 2003). In plants, however, despite the large proportion of the clustered LTR elements in their genomes, direct evidence is missing for targeted integration of LTR elements into gene-poor regions. 
Several plant LTR retrotransposons have been shown to be mobile, such as tobacco Tnt1 and Tto1, rice Tos17, and lotus $L O R E 1$, but all of them retrotranspose into genic regions, and no preferential integration into gene-poor regions has been reported for these plant retrotransposons (Hirochika et al. 1996; Okamoto and Hirochika 2000; Courtial et al. 2001; Yamazaki et al. 2001; Madsen et al. 2005; Le et al. 2007; Hou et al. 2010).

Precise transposon sequences throughout the genome of Arabidopsis thaliana make it an ideal model organism to analyze the behavior of transposons at the sequence level (The Arabidopsis Genome Initiative 2000; Le et al. 2000; Peterson-Burch et al. 2004). The genome of $A$. thaliana, as many other plant species, contains large clusters of LTR retrotransposons around centromeres. They are localized in regions flanking the cores of centromeres, which are composed of tandemly arrayed satellite repeats of 178 base pairs (bp) (Heslop-Harrison et al. 2003). The most abundant and most well-characterized subfamily of the pericentromeric LTR retrotransposons of A. thaliana is called Athila (Pelissier et al. 1995; Steimer et al. 2000; Peterson-Burch et al. 2004; Slotkin 2010). However, no mobile Athila has been identified so far.

Using $A$. thaliana, we and others recently reported identification of multiple families of mobile LTR retrotransposons (Mirouze et al. 2009; Tsukahara et al. 2009). These retrotransposons-ATGP3, ATCOPIA13, ATCOPIA21, and ATCOPIA93 (also called Evade)—are silent in the wild type, but are activated when their DNA methylation is abolished in mutants of a chromatin remodeling factor gene, $D D M 1$ (decrease in DNA methylation 1) (Vongs et al. 1993; Jeddeloh et al. 1999). Unlike Athila, these mobile elements are low copy number in the genome of $A$. thaliana. In addition, examination of integration specificity for two of them, ATCOPIA93 and ATGP3, revealed that they did not show targeted integration into gene-poor regions (Mirouze et al. 2009; Tsukahara et al. 2009). Interestingly, however, a closely related Arabidopsis species, Arabidopsis lyrata, has high-copy-number retrotransposons related to ATCOPIA93, and their sequence similarity suggests recent proliferation (Tsukahara et al. 2009|. Furthermore, they are localized within centromeric repeats. Thus, we suspected that these copies in $A$. lyrata might have the ability to retrotranspose preferentially into the centromeres.

In this study, we directly examined the mobility of one of the ATCOPIA93-related elements in A. lyrata, which we named Tal1 (Transposon of Arabidopsis lyrata 1). Tal1 was mobile in the genome of $A$. thaliana and showed targeted integration into centromeric repeats, demonstrating the first example of targeted integration of a plant retrotransposon into gene-poor regions. Direct characterization of integration specificities of these mobile elements is revealing unexpectedly dynamic controls for evolution of the heterochromatin and repeats.

\section{Results}

\section{Tall was mobile in transgenic A. thaliana plants}

We showed previously that multiple copies of $A$. lyrata elements related to ATCOPIA93 are localized in centromeric repeats (Tsukahara et al. 2009). On the other hand, the mobile ATCOPIA93 copy (At5g17125) is localized in the arm region of the $A$. thaliana genome; and this element frequently retrotransposes into chromosomal arm regions (Mirouze et al. 2009). The apparent difference in genomic distributions of these COPIA93-like elements in A. thaliana and $A$. lyrata could be due to differences in host environments; for example, centromeric satellite sequences are $\sim 30 \%$ different between these two species (Kawabe and Nasuda 2005). Alternatively, the difference may reflect differences in the properties of the transposons. To test these possibilities directly, we introduced one of the $A$. lyrata copies into the $A$. thaliana genome by transformation.

We first tried to select a mobile $A$. lyrata copy. The $A$. lyrata genome contains at least 16 copies of elements similar to ATCOPIA93. The identities of the $5^{\prime}$ and $3^{\prime}$ LTRs of these elements are $99.2 \%-100 \%$, suggesting that their proliferations are recent. For transformation, we chose an $A$. lyrata element with $100 \%$ identity of the $5^{\prime}$ and $3^{\prime}$ LTRs and with an ORF having the potential to encode functional proteins. That copy, which we named Tal1, was amplified by PCR from the A. lyrata genome, cloned into the pRI909 binary vector (Fig. 1A), and introduced into $A$. thaliana by Agrobacterium-mediated transformation. Because Tal1 may be silenced by DNA
A

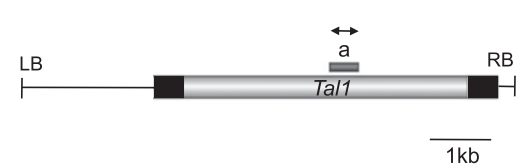

B

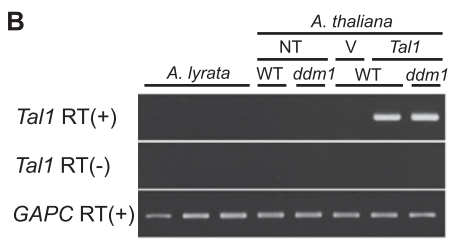

C

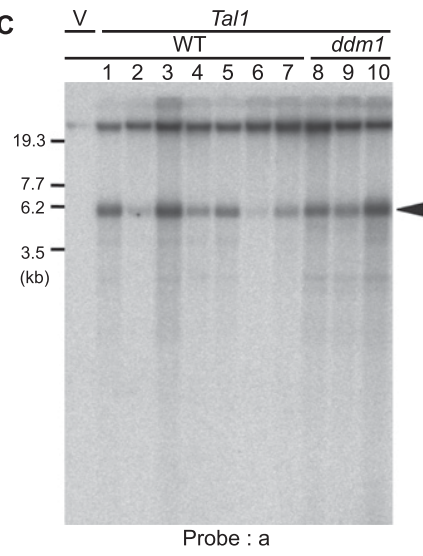

Figure 1. Activity of Tal1 in transgenic A. thaliana plants. $(A)$ The structure of T-DNA around Tal1 in pRI909 binary vector. Black boxes indicate $5^{\prime}$ and $3^{\prime}$ LTRs of Tal1. (B) Transcription of Tal1 examined by RT-PCR. The region amplified by PCR is shown with a black line with two arrowheads in $A$. T1 transgenic plants were used. GAPC was used as a positive control. Lack of Tal1 transcript in the original host A. lyrata is associated with their high level of DNA methylation (not shown). (C) Southern blot analysis using undigested genomic DNA of independent lines with the Tal1 transgene (T2 generation). The top bands represent undigested chromosomal DNA. The bottom bands correspond to the length of extrachromosomal linear DNA of Tal1 (black arrowhead). The probe is shown in $A$ with a gray bar. (NT) Plant not transformed; (V) plant transformed with vector. 
methylation, we used both wild-type and $d d m 1$ mutant plants for the host. The $d d m 1$ mutation is known to release transcriptional silencing of diverse methylated transposons (Hirochika et al. 2000; Miura et al. 2001; Singer et al. 2001; Lippman et al. 2004; Mirouze et al. 2009; Tsukahara et al. 2009).

In transgenic plants of both wild type and $d d m 1$, we could detect the transcript of Tal1 (Fig. 1B). Generally, during retrotransposition of an LTR element, RNA for the element is reverse-transcribed to produce linear extrachromosomal DNA, which subsequently integrates into the host genome. We examined the presence of the extrachromosomal DNA in the transgenic plant lines by Southern blotting of genomic DNA not digested by restriction enzymes. In every transgenic line examined, we could detect the extrachromosomal Tal1 DNA of the expected length (Fig. 1C).

We next examined Tal1 retrotransposition by Southern analyses using genomic DNA from multiple transgenic lines. After digestion by EcoRV, membranes were first hybridized with a probe for the T-DNA vector region flanking Tal1. By hybridization to that probe, a common signal of the expected length was detected in each transgenic line (Fig. 2B). When the same membranes were rehybridized with a probe for Tal1, additional signals were detected (Fig. 2B), suggesting the presence of Tal1 DNA in these transgenic plants outside of the original copy introduced by T-DNA.

The signal specifically seen by the Tal1 probe had a common pattern among these transgenic lines; the common strong signals were observed around $2.0-\mathrm{kb}$ and highmolecular-weight positions (>19 kb) (Fig. 2B). The signals observed around $2.0 \mathrm{~kb}$ match to the expected size of the linear extrachromosomal DNA digested by EcoRV (Fig. 2B, arrowheads). The other strong signals with high molecular weight imply that Tal1 is located within DNA sequences apart from EcoRV sites. We also used another restriction enzyme, HindIII, and compared the patterns of signals (Fig. 3). In addition to signals expected for extrachromosomal DNA digested by HindIII (653 bp and 498 bp), multiple signals were detected in low-molecular-weight regions (500 800 bp), suggesting de novo Tal1 insertion near HindIII sites. Taken together, these results of Southern analyses suggest that a common feature of Tal1
A

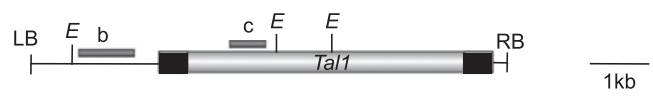

B
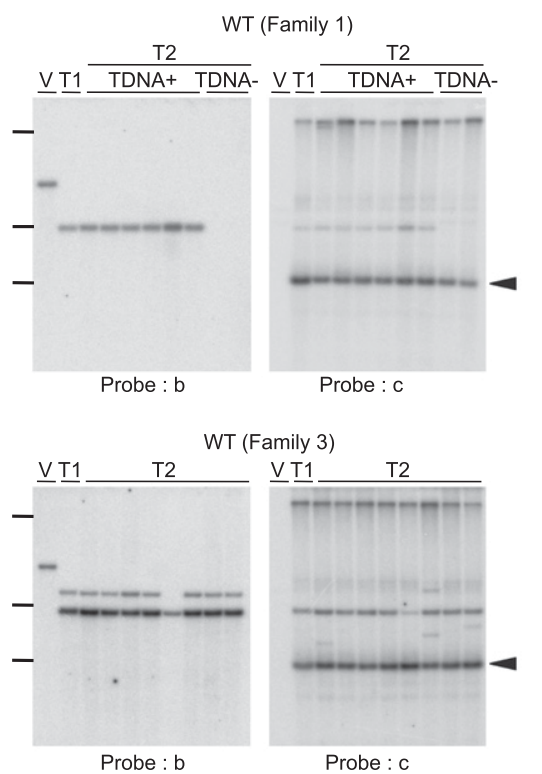

$d d m 1$ (Family 8)

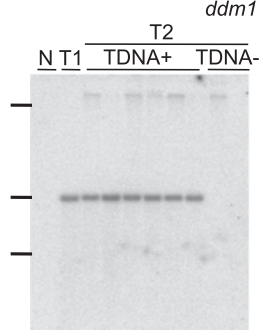

Probe : $b$

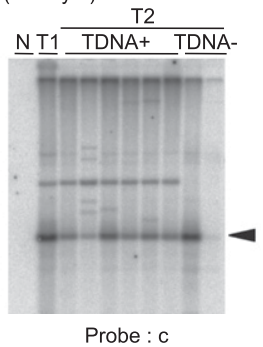

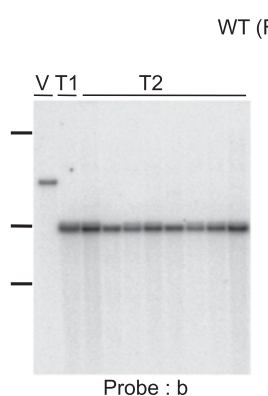

WT (Family 2)
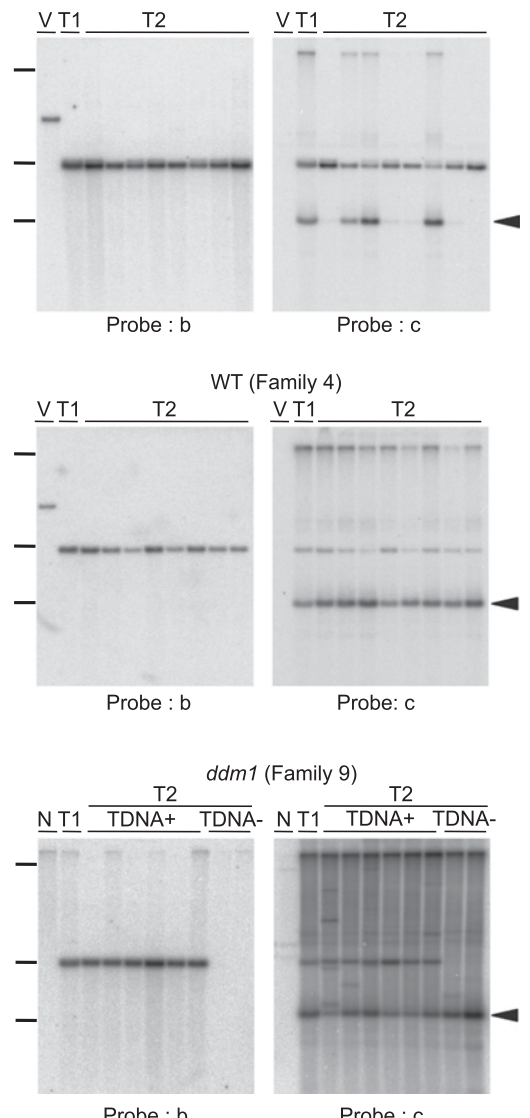

Probe : $b$

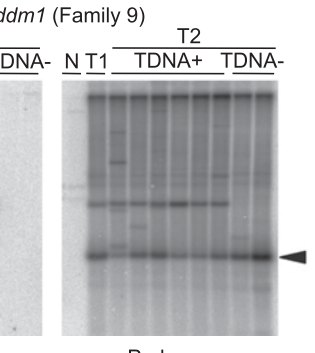

Probe : c
Figure 2. Retrotranspositions of Tal1 in transgenic plants. (A) EcoRV sites (E) and probe positions (gray bars) in the T-DNA region. $(B)$ Six independent transgenic lines (four wild-type lines and two $d d m 1$ lines) were characterized. Their family numbers correspond to the lane numbers in Figure 1C (the T2 plants were siblings of the same family). DNA from T1 and T2 plants were digested by EcoRV, which is insensitive to DNA methylation, and were hybridized with a probe for T-DNA (probe b) and, subsequently, with a probe for Tal1 (probe c). The position expected for the extrachromosomal DNA fragment is indicated by a black arrowhead. The Tal1 probe does not hybridize to endogenous COPIA93 family retrotransposons in $A$. thaliana. The additional band seen by probe $\mathrm{b}$ in family 3 is likely to reflect partial or rearranged T-DNA integration. In each family, 14 individuals of T2 plants were checked by PCR for the presence of T-DNA, and eight of the plants were used in the Southern blot. For families 1, 8, and 9, we could find T2 plants without T-DNA (T-DNA-), and two of them were included in each family. Note that some of them have signals for the extrachromosomal DNA, suggesting that newly integrated copies have activity for retrotransposition. The lengths of the molecular weight markers are $19.33 \mathrm{~kb}$, $3.47 \mathrm{~kb}$, and $1.88 \mathrm{~kb}$. (N) Plant not transformed. 
A

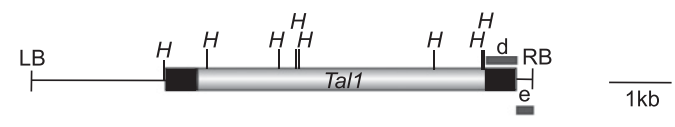

B
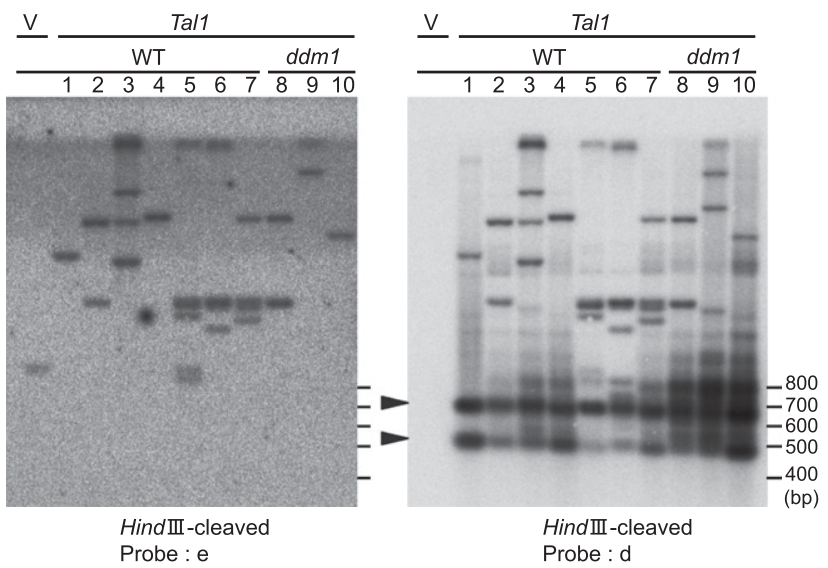

Figure 3. Retrotranspositions of Tal1 near HindIII sites. (A) HindIII sites $(\mathrm{H})$ and probe positions (gray bars). (B) T2 plants, siblings of the transgenic lines used in Figure 1, were examined first with probe e for the vector and subsequently with probe $\mathrm{d}$ for Tal1. The position expected for the extrachromosomal DNA fragment is indicated by a black arrowhead.

integration sites in these lines is loci near HindIII sites and apart from EcoRV sites. We suspected that these might indicate insertions of Tal1 in centromeric tandem arrays, which normally includes HindIII sites, but rarely includes EcoRV sites.

\section{Tall preferentially integrates into $178-b p$ centromeric repeats}

To directly examine Tal1 insertion site specificity, we performed whole-genome resequencing of the transgenic plants (two wild-type and two $d d m 1$ lines in the $\mathrm{T} 2$ generation, one individual plant for each line) using an Illumina Genome Analyzer IIx sequencer. Eighty million to 110 million reads (read length: $110 \mathrm{bp}$ ) were generated for each line, covering $>50 \times$ of the genomes. In order to detect flanking sequences of Tal1, we first screened reads containing $30 \mathrm{bp}$ of sequence for the $5^{\prime}$ or 3' end of Tal1. From these reads, we then subtracted hits for the T-DNA vector, which correspond to the original transgene, and internal regions of Tal1 flanking the LTRs, which reflect duplication of the LTR sequence. Remaining reads containing $>20$ nucleotides (nt) were used for identifying new integration sites of Tal1. The numbers of such reads were 46 and 758 for two lines of wild type, and 1471 and 2039 for two lines of $d d m 1$ mutants.

We then examined how the flanking sequence of each read matches the consensus sequence of the centromeric satellite repeats. For each read, the proportion of the identities of the flanking $20 \mathrm{nt}$ was examined along the 178-nt centromeric satellite unit, and the maximum value was used for further analysis. Distributions of such proportions were generally bimodal (Fig. 4; Supplemental Fig. S1), reflecting sequences within the satellites and other regions. For randomly chosen genomic reads (Fig. 4B,D,F), $\sim 8 \%$ corresponded to the satellites, which is consistent with previous estimates for the amount of centromeric satellites (The Arabidopsis Genome Initiative 2000; Hosouchi et al. 2002).

When reads flanking the new Tal1 integrations were analyzed for the two wild-type lines, 43 out of $46(93 \%)$ and 721 out of $758(95 \%)$ reads belong to the population for the satellites (Fig. 4A; Supplemental Fig. S1). Thus the new Tal1 integrations were biased strongly toward the satellites. In addition, many of the other reads (one out of three and 36 out of 37) represent nested integration into another Tal1 (Supplemental Fig. S2). The strong bias toward the satellite was also found in the $d d m 1$ background. In $d d m 1$ lines, 1394 out of $1471(95 \%)$ and 1794 out of $2039(88 \%)$ reads correspond to the integrations into the satellite (Fig. 4C; Supplemental Fig. S1), and nested integration into another Tal1 was also found (17 out of 77 and 39 out of 245) (Supplemental Fig. S2). Integration sites of Tal1 were distributed throughout the satellite unit in both wild type and $d d m 1$ (Fig. 5A; Supplemental Table S1), although local hot and cold spots for integration exist (Fig. 5B; Supplemental Table S2).

We showed previously that ATCOPIA93 of A. thaliana, which is related to Tal1 of A. lyrata, proliferates in the $d d m 1$ background. We then examined the integration sites of ATCOPIA93 in five independent $d d m 1$ lines. The distribution of new integration of ATCOPIA93 was not biased toward the satellites (Fig. 4E; Supplemental Fig. S1). When compared with random reads from the same individual, the ATCOPIA93 integration sites were even biased toward the opposite direction; their integrations into the satellites were 11 out of $634(2 \%), 95$ out of $2823(3 \%)$, one out of $249(0.4 \%), 182$ out of 5990 (3\%), and 104 out of 4644 $(2 \%)$, which are significantly lower than those of random reads (Fig. 4E,F; Supplemental Fig. S1). This observation is consistent with a previous report showing frequent integrations of ATCOPIA93 into chromosomal arm regions (Mirouze et al. 2009). Even though ATCOPIA93 and Tal1 are highly related, their targeting specificities differ considerably.

Sequences of COPIA93/20 of A. lyrata suggest that their recent burst of integrations into centromeres occurred in independent subfamilies

The results above suggest that Tal1 and the mobile ATCOPIA93 (At5g17125) have different integration site specificities; the former specifically integrates into centromeric repeats, while the latter do not. In order to know which is the ancestral property, we extended the sequence analyses to similar elements in the genomes of $A$. thaliana and A. lyrata. Sequence comparison of $A$. lyrata copies (shown by red lines in Fig. 6) suggests that proliferation of these copies occurred in four clusters of sequences independently. On the other hand, only five 

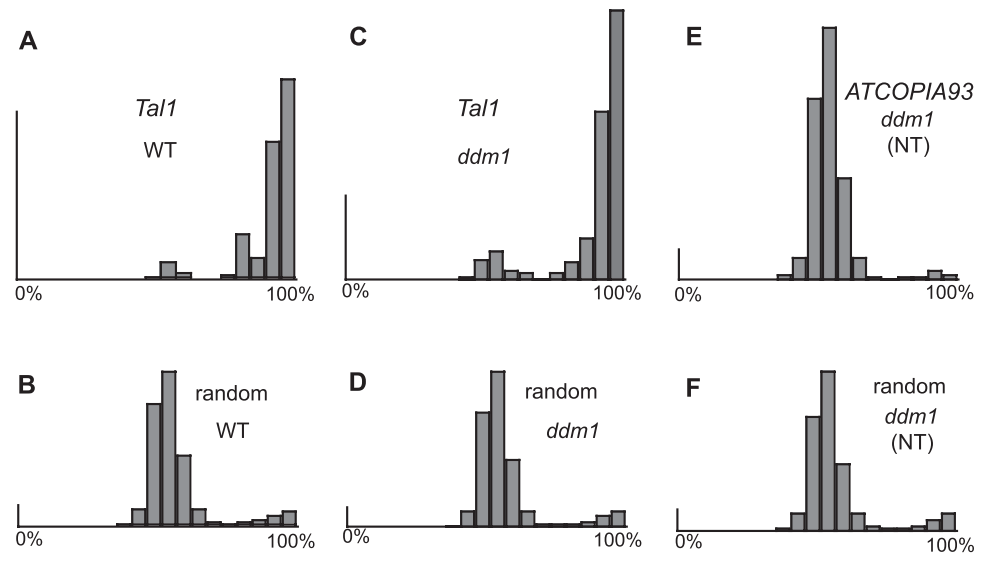

Figure 4. Integration of Tal1 in centromeric repeats and unbiased integration of ATCOPIA93. Integration sites of Tal1 and ATCOPIA93 were examined by wholegenome resequencing. Proportions of matches to the consensus sequence of the centromeric satellite are shown for reads flanking transposon insertions. $(A, C)$ Flanking sequences for new integration sites of Tal1. Results for one wild-type and one $d d m 1$ transgenic line are shown. (E) Integration sites of ATCOPIA93 examined in the same manner for one nontransgenic (NT) $d d m 1$ line self-pollinated eight times (Kakutani et al. 1996) (DNA from multiple sibling plants were pooled for the nontransgenic ddm1 sample). $(B, D, F)$ About 6000 random genome reads were analyzed for each individual used in $A, C$, and $E$. For each class, results of individual with maximum number of reads are shown in this figure. Supplemental Figure S1 shows the results with additional plants: Tal1 insertions in four transgenic lines (two ddm 1 and two wild type), and ATCOPIA93 insertions in five independently self-pollinated $d d m 1$ lines. In each panel, the vertical bar represents 300 reads. The consensus sequence of the centromeric satellite used is AASCTTCTTCTTGCTTCTCAAAGCTTTSATGGTGTAGCCAAAGTCCRTATGAGTCTTTGKCT TTGTRTCTTCTAACAAGGAWACAMTACTTAGGCTTTTARGATCVGGTTGCGGTTYWAGTTCTTATACTCAATCATACACA TGACATCWAGTCATATTYGACTCCAAAACACTAACC, with V representing A, C, or G; W representing A or T; R representing A or $\mathrm{G}$; $\mathrm{Y}$ representing $\mathrm{C}$ or $\mathrm{T}$; $\mathrm{K}$ representing $\mathrm{G}$ or $\mathrm{T}$; $\mathrm{M}$ representing $\mathrm{A}$ or $\mathrm{C}$; and $\mathrm{S}$ representing $\mathrm{C}$ or $\mathrm{G}$. Integration sites other than centromeric satellites are shown in Supplemental Table S1.

copies of A. thaliana elements were found. We showed previously that $A$. lyrata elements related to mobile ATCOPIA93 (cluster 1 in Fig. 6, which includes Tal1) are localized in centromeric repeats (Tsukahara et al. 2009). We then examined whether $A$. lyrata copies in other clusters (clusters 2, 3, and 4) are localized in centromeres.

In total, 288 unique $A$. lyrata loci of COPIA93/20 were characterized. Among them, 272 have internal regions, and 16 are solo LTRs. In all of the four clusters, most copies are flanked by centromeric satellite sequences $172 \%$ in total) (Fig. 7). About $4 \%$ of loci are flanked by unknown sequences, but centromeric satellite sequences exist within $500 \mathrm{bp}$. In addition, most of the integrations into nonsatellite regions are flanked by other transposons (Fig. 7). The majority of these transposons belong to COPIA93/20 families or Athila, which are likely to be localized in pericentromeric or centromeric regions (Pelissier et al. 1995). Among the 288 loci examined, only six loci had flanking sequence with homology with genic and intergenic regions of $A$. thaliana. Almost all $195 \%$, even if flanking sequences without detectable homology were excluded) integrations are likely to be localized within centromeric or pericentromeric nongenic regions. These trends of integration site bias were found in all four clusters of $A$. lyrata elements (Fig. 7). These results suggest that the preferential accumulation in centromeric satellites is the ancestral property of these elements before separation of the mobile ATCOPIA93 and Tal1.

\section{Discussion}

\section{Centromere-specific targeted integration of Tal1}

In this study, we showed that Tal1 integrated almost exclusively into centromeric repeats of $A$. thaliana. One could argue that even though the de novo Tal1 integrations were found in centromeres, the possibility remains that it is due to natural selection; cells or individuals with Tal1 insertion into gene-rich regions may be immediately eliminated by natural selection. We think that possibility is unlikely for multiple reasons. First, centromeres are

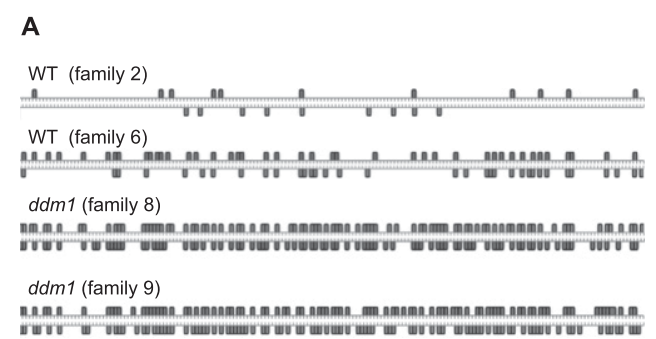

B

\begin{tabular}{|l|c|c|}
\hline & forward plus & forward minus \\
\hline reverse plus & $93(69.8)$ & $19(42.2)$ \\
\hline reverse minus & $18(41.2)$ & $48(24.8)$ \\
\hline
\end{tabular}

Figure 5. Distribution of Tal1 integration sites within the 178bp centromeric repeat of A. thaliana. (A) Positions of Tal1 integrations. The 178 positions are those in the sequences shown in the legend for Figure 4. The top and bottom bars indicate insertions with $5^{\prime}$-to-3' and $3^{\prime}$-to-5' directions of Tal1, respectively. The target site duplication of Tal1 was normally 5 bases long, and the third nucleotide from the integration site is shown because that site would be the same for the $5^{\prime}$ and $3^{\prime}$ flanking sequences. The number of reads for each site is shown in Supplemental Table S2. (B) The 178 sites were divided into four classes depending on the presence or absence of Tal1 insertion in forward $\left(5^{\prime}\right.$-to- $\left.3^{\prime}\right)$ and reverse $\left(3^{\prime}\right.$-to- $\left.5^{\prime}\right)$ orientations in two $d d m 1$ transgenic lines in $A$. The numbers in parentheses represent the values expected if insertions in forward and reverse orientations are independent. The skew $\left(P<10^{-12}\right)$ suggests positive association and the presence of local hot and cold spots for Tal1 insertions within the satellite unit. 


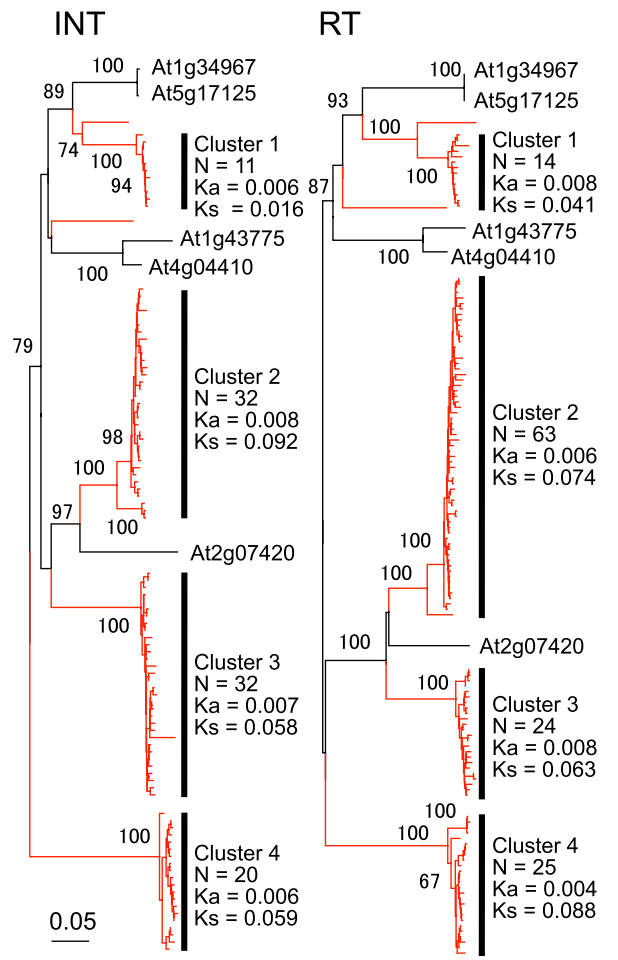

Figure 6. Phylogenetic relationship of COPIA93/20 elements of $A$. thaliana and A. lyrata. The phylogenetic trees were made based on the nucleotide sequences of integrase (INT) and reverse transcriptase (RT) domains. A. lyrata copies are shown by red lines, and $A$. thaliana copies are shown by black lines. At2g07420 has been annotated as ATCOPIA20, and four other A. thaliana copies have been annotated as ATCOPIA93. Each of the A. lyrata clusters includes $\sim 10-60$ copies with an average synonymous divergence of $2 \%-10 \%$, suggesting recent bursts. As $>100$ shotgun reads were sometimes found with identical sequences for the integrase and reverse transcriptase domains $(\sim 600 \mathrm{bp})$, some of them may represent multiple copies with identical sequences, reflecting even more recent bursts. Phylogenetic trees were constructed by the neighbor-joining method using distances corrected by the Jukes-Cantor method. Bootstrap probabilities were estimated by 500 replicates and are shown only in major branches. The number of sequences and the average number of nucleotide differences (both synonymous and replacement sites) in each cluster are shown at the right of the cluster.

not the only gene-poor regions in the genome; if exclusion from gene-rich regions is the main force to cause the biased distribution, Tal1 would also accumulate in other gene-poor regions, such as ribosomal DNA and intergenic regions, especially in the early phase of the selection. That was not the case (Fig. 4; Supplemental Fig S1; Supplemental Table S1). Second, we could not detect any sign of elimination of cells or individuals in the Tal1 transgenic plants. The transgenic lines were morphologically normal even after multiple rounds of self-pollinations where Tal1 continues to be expressed (Supplemental Fig. S3), and their fertility was indistinguishable from that of the control plants. Finally, ATCOPIA93, a copy closely related to Tal1, did not show biased accumulation in centromeres; many of the insertions were found in genic regions (Fig. 4E; Supplemental Fig. S1). It is hard to speculate that the deleterious effects of genic insertion of ATCOPIA93 are much weaker than those of Tal1, because their structures are similar (Fig. 6). The accumulation of Tal1 in centromeres is likely due to targeted integration, rather than natural selection.

What feature in centromeres does Tal1 recognize? The centromeric satellite sequences are known to evolve fast (Mallik and Henikoff 2002). Although A. lyrata and A. thaliana are closely related species, divergence of the satellite sequences is $\sim 30 \%$ (Kawabe and Nasuda 2005). Still, Tal1 targeted to centromeres of A. thaliana. Instead of the primary sequence, the higher-order chromatin configuration, unique to centromeres, may be recognized by Tal1. Centromeric and pericentromeric regions tend to have a condensed structure, which is marked by DNA methylation and dimethylation of histone H3 Lys 9, and the $d d m 1$ mutation abolishes the condensed structure and the modifications (Gendrel et al. 2002; Soppe et al. 2002; Lippman et al. 2004). However, even in the $d d m 1$ mutant, most of the insertions of Tal1 were found in centromeric repeats, suggesting that marks of centromeres

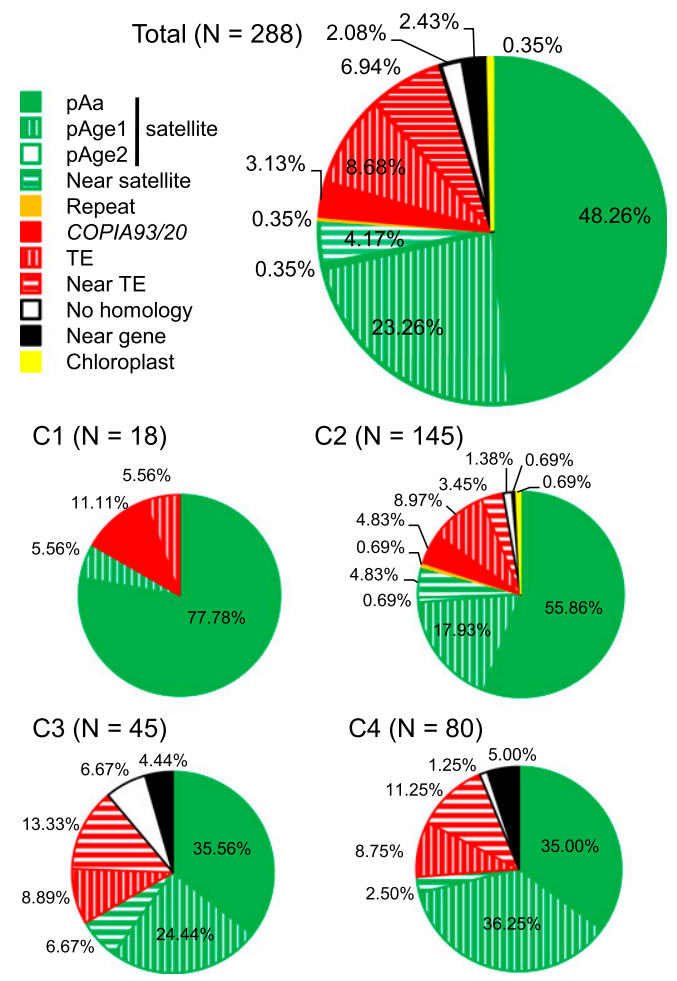

Figure 7. Genomic localization of A. lyrata COPIA93/20 elements. Proportions of inserted region categories are shown for the total and each of four clusters (C1-C4). pAa, pAge1, and pAge2 are centromeric satellite sequences in A. lyrata. Integrations into the COPIA93/20 family were separated from those into the other transposable element (TE) families. If centromeric satellites (either pAa, pAge1, or pAge2) or TE sequences were found within $500 \mathrm{bp}$ from the insertion site, the loci were categorized as "Near" them. 
recognized by Tal1 do not depend on DDM1 function. Centromeres are also marked by specific proteins (Kanizay and Dawe 2009), which could be more likely candidates for the target of Tal1 integration.

\section{Evolutionary dynamics of COPIA93/20 elements} and centromeric satellites in $\mathrm{A}$. lyrata and $\mathrm{A}$. thaliana

A notable feature suggested from the sequence data of COPIA93/20 elements shown in Figure 6 is that their bursts occurred in four independent lineages in A. lyrata, while their possible orthologous copies in A. thaliana remained low copy. The lyrata-specific bursts are also found in other subfamilies of LTR elements (Hu et al. 2011), consistent with the theoretical prediction that active transposons propagate more easily in outcrossing $A$. lyrata than in self-pollinating $A$. thaliana (Hickey 1982).

The phylogenetic analysis also suggests that targeting to centromeres is likely an ancestral property of $A$. lyrata elements, which is lost in the mobile ATCOPIA93 (At5g17125). Interestingly, however, none of the other four copies of $A$. thaliana elements shown in Figure 6 (Atlg34967, Atlg43775, At4g04410, and At2g07420) localize in centromeric satellites either. Considering that, it is especially surprising that Tal1 has an ability to target to centromeric satellite sequences of $A$. thaliana (this study), even though no copy is found to localize in satellite sequences in the natural wild-type $A$. thaliana.

It is enigmatic that no COPIA93/20 elements are localized in centromeric satellites in A. thaliana. One possible explanation is that $A$. thaliana has lost the typical ancestral COPIA93/20 copies within centromeric satellites. A transposon insertion within the satellite repeat could be lost by unequal crossing over. In addition, as the karyotype of $A$. thaliana is different from those in other related species, its centromeric sequences seem to have been replaced by the current form in the recent past (Kawabe et al. 2006). During such replacement, COPIA93/ 20 localized in the centromeric satellites may have been lost, and only copies outside the satellites may have been transmitted.

In any case, the evolution of integration specificity of the retroelements plays a key role in heterochromatin dynamics. Generally, targeted integration of a retroelement depends on recognition of the target site by its integrase (Sandmeyer 1990; Bushman 2003; Gao et al. 2008). Amino acid sequences of integrases of Tal1 and ATCOPIA93 are $>90 \%$ identical (Supplemental Fig. S4). If the difference in the targeting specificities is due to their structural difference, the responsible motifs may be clarified by examination of targeting specificity after generating retrotransposon constructs with chimeric integrases.

\section{Perspectives}

Accumulation of LTR retrotransposons around centromeric satellite repeats is a general feature found in many plant species. For example, LTR retrotransposons such as maize CRM or rice CRR are localized around centromeres and have been extensively investigated on the levels of sequence, chromosomal location, and evolution
(Cheng et al. 2002; Nagaki et al. 2005; Ma and Jackson 2006; Du et al. 2010). However, no mobile copy of the centromeric LTR element had been reported to date. Tal1 might provide an experimental system for understanding the effects of retrotransposon insertion de novo for the evolution of centromeres.

Retroelements have been widely used for transformation vectors in human gene therapies. Especially useful vectors are those with specific integration targets (Bushman 2003). However, as far as we know, no mobile element targeting to centromeres has previously been identified in any organism. Tal1 might provide a useful vector to engineer chromosome organization, for example, by introducing loxP sites into centromeres to shuffle chromosome arms by CRE recombinase or introducing a telomere construct (Yu et al. 2007) to make telocentric chromosomes.

\section{Materials and methods}

\section{Plant materials}

The wild-type and $d d m 1$ mutant of $A$. thaliana plants used were in a Col background. The mutant allele $d d m 1-1$ (Vongs et al. 1993) was used throughout.

\section{Construction of the pRI909 vector containing Tall and transformation}

A PstI-SacI fragment of the $5^{\prime}$ half of Tal1 and a SacI-EcoRI fragment of the $3^{\prime}$ half of Tal1 were amplified from genomic DNA of A. lyrata by nested PCR using the primers shown in Supplemental Table S3. These fragments were cloned into the PstI-EcoRI site of the pRI909 vector (Takara) by Mighty Mix (Takara). A. thaliana wild type and $d d m 1$ mutants were transformed by the standard floral dip method (Clough and Bent 1998) using a pRI909 vector containing Tal1. The T-DNAs transferred to $A$. thaliana carried a neomycin phosphotransferase gene (NPTII) that was used for the selection marker.

\section{Southern analysis}

Genomic DNA of $A$. thaliana was extracted from mature leaves using Nucleon PhytoPure genomic DNA extraction kits (GE Healthcare). Probes for Tal1 were amplified using the primers described in Supplemental Table S3.

\section{$R T-P C R$}

For RT-PCR, total RNA was extracted from leaf tissue using the SV Total RNA Isolation system (Promega) and was treated with DNase I (Promega). From $2 \mu \mathrm{g}$ of total RNA, cDNA was synthesized using the First Strand cDNA Synthesis kit (GE Healthcare) and a $\mathrm{pd}(\mathrm{N}) 6$ primer. A one-tenth portion of the $\mathrm{RT}$ reaction was used as a template for PCR (total, $10 \mu \mathrm{L}$ ). PCR conditions were $3 \mathrm{~min}$ at $94^{\circ} \mathrm{C} ; 25-33$ cycles of $30 \mathrm{sec}$ at $94^{\circ} \mathrm{C}, 30 \mathrm{sec}$ at $55^{\circ} \mathrm{C}$, and $60 \mathrm{sec}$ at $72^{\circ} \mathrm{C}$; and $3 \mathrm{~min}$ at $72^{\circ} \mathrm{C}$. The PCR product was then separated by electrophoresis on a $1.5 \%$ agarose gel. Primer pairs for RT-PCR are shown in Supplemental Table S3.

\section{Whole-genome resequencing}

Tal1 integration sites were analyzed in two wild-type and two $d d m 1$ lines, which are siblings of plants in lanes $2,6,8$, and 9 in 
Figure 1C. The results of the four lines are included in Supplemental Figure S1, but Figure 4 includes only the results for two of them, corresponding to lanes 6 and 9. Whole-genome resequencing libraries (insert size: 200-350 bp) were prepared using a Paired End DNA Sample Prep kit (Illumina). The quality and quantity of DNA samples were measured using the QuantiT PicoGreen dsDNA Assay kit (Life Technologies), Agilent 2100 Bioanalyzer, and Power SYBR Green PCR Master mix (Applied Biosystems). The genomic libraries were used to generate clusters on the Illumina cBot using the Paired End Cluster Generation kit (Illumina) and were sequenced on the Illumina Genome Analyzer IIx and HiSeq2000 sequencers with 111 and 101 cycles, respectively. Sequences and quality scores passing through the standard Illumina pipeline filters were retained for further analysis. Raw sequence data for the transgenic plants were deposited in the DDBJ (DNA Data Bank of Japan) Sequence Read Archive (DRA; accession nos. DRA000394, 000425-000427). Flanking sequences of transposons were identified by simply extracting reads containing terminal regions of transposons, using each of the paired end sequences independently. When $20 \mathrm{nt}$ of Tal1 flanking sequence was analyzed, a sequence with $\geq 80 \%$ match to the centromeric satellite consensus was categorized as flanked by the satellite. When the $20 \mathrm{nt}$ of flanking sequence matched $100 \%$ to a part of the Tal1 sequence, it was classified as a nested integration into another Tal1. The integration site specificity of Tal1 was also examined by a suppression PCR technique (Tsukahara et al. 2009). By that method, 30 out of 33 detected Tal1 flanking sequences were found to match the centromeric satellites (data not shown), consistent with the genome resequencing results.

\section{Analysis of A. lyrata genome sequence data}

Transposon sequences were obtained from A. lyrata shotgun genome sequence data. Integrase or reverse transcriptase domain sequences were used as queries to a BLAST search at the NCBI Trace Archive (http://www.ncbi.nlm.nih.gov/Traces/trace.cgi?). The obtained shotgun reads were aligned, and sequences with two or more identical reads were retained. From these conserved domain sequences, a sequence walk was done to extend alignment to the end of the transposon sequence. The LTR sequence was defined by comparing flanking sequences of different loci sequences to determine both $5^{\prime}$ and $3^{\prime}$ ends. The LTR sequences were used for queries to obtain other loci simultaneously. Sequences flanked to LTR regions were checked by the NCBI BLAST homepage restricted to Arabidopsis sequence entries.

\section{Acknowledgments}

We thank Akiko Terui for technical assistance, and Yasushi Hiromi and Tetsusi Iida for critical comments on the manuscript. This study was supported by a fellowship by the Japan Society for the promotion of Science to S.T. and grants from the Mitsubishi Foundation, the Takeda Science Foundation, and the Japanese Ministry of Education, Culture, Sports, Science, and Technology (19207002 and 19060014) to T.K.

\section{References}

The Arabidopsis Genome Initiative. 2000. Analysis of the genome sequence of the flowering plant Arabidopsis thaliana. Nature 408: 796-815.

Bushman FD. 2003. Targeting survival: Integration site selection by retroviruses and LTR-retrotransposons. Cell 115: 135-138.
Cheng Z, Dong F, Langdon T, Ouyang S, Buell CR, Gu M, Blattner FR, Jiang J. 2002. Functional rice centromeres are marked by a satellite repeat and a centromere-specific retrotransposon. Plant Cell 14: 1691-1704.

Clough SI, Bent AF. 1998. Floral dip: A simplified method for Agrobacterium-mediated transformation of Arabidopsis thaliana. Plant J 16: 735-743.

Courtial B, Feuerbach F, Eberhard S, Rohmer L, Chiapello H, Camilleri C, Lucas H. 2001. Tnt1 transposition events are induced by in vitro transformation of Arabidopsis thaliana, and transposed copies integrate into genes. Mol Genet Genomics 265: 32-42.

Dawe RK, Hiatt EN. 2004. Plant neocentromeres: Fast, focused, and driven. Chromosome Res 12: 655-669.

Du J, Tian Z, Hans CS, Laten HM, Cannon SB, Jackson SA, Shoemaker RC, Ma J. 2010. Evolutionary conservation, diversity and specificity of LTR-retrotransposons in flowering plants; insights from genome-wide analysis and multispecific comparison. Plant J 63: 584-598.

Gao X, Hou Y, Ebina H, Levin HL, Voytas DF. 2008. Chromodomains direct integration of retrotransposons to heterochromatin. Genome Res 18: 359-369.

Gendrel AV, Lippman Z, Yordan C, Colot V, Martienssen RA. 2002. Dependence of heterochromatic histone H3 methylation patterns on the Arabidopsis gene DDM1. Science 297: 1871-1873.

Grewal SI, Jia S. 2007. Heterochromatin revisited. Nat Rev Genet 8: $35-46$.

Hawkins JS, Kim H, Nason JD. 2006. Differential lineagespecific amplification of transposable elements is responsible for genome size variation in Gossypium. Genome Res 16: 1252-1261.

Heslop-Harrison IS, Brandes A, Schwarzacher T. 2003. Tandemly repeated DNA sequences and centromeric chromosomal regions of Arabidopsis species. Chromosome Res 11: 241-253.

Hickey DA. 1982. Selfish DNA: A sexually-transmitted nuclear parasite. Genetics 101: 519-531.

Hirochika H, Sugimoto K, Otsuki Y, Tsugawa H, Kanda M. 1996. Retrotransposons of rice involved in mutations induced by tissue culture. Proc Natl Acad Sci 93: 77837788.

Hirochika H, Okamoto H, Kakutani T. 2000. Silencing of retrotransposons in Arabidopsis and reactivation by the ddm1 mutation. Plant Cell 12: 357-369.

Hosouchi T, Kumekawa N, Tsuruoka H, Kotani H. 2002. Physical map-based sizes of the centromeric regions of Arabidopsis thaliana chromosomes 1, 2, and 3. DNA Res 9: $117-121$.

Hou Y, Rajagopal J, Irwin PA, Voytas DF. 2010. Retrotransposon vectors for gene delivery in plants. Mob DNA 1: 19. doi: 10.1186/1759-8753-1-19.

Hu TT, Pattyn P, Bakker EG, Cao J, Cheng JF, Clark RM, Fahlgren N, Fawcett JA, Grimwood J, Gundlach H, et al. 2011. The Arabidopsis lyrata genome sequence and the basis of rapid genome size change. Nat Genet 43: 476-481.

Jeddeloh JA, Stokes TL, Richards EJ. 1999. Maintenance of genomic methylation requires a SWI2/SNF2-like protein. Nat Genet 22: 94-97.

Kakutani T, Jeddeloh JA, Flowers S, Munakata K, Richards EJ. 1996. Developmental abnormalities and epimutations associated with DNA hypomethylation mutations. Proc Natl Acad Sci 93: 12406-12411.

Kanizay L, Dawe RK. 2009. Centromeres: Long intergenic spaces with adaptive features. Funct Integr Genomics 9: 287-292. 
Kawabe A, Nasuda S. 2005. Structure and genomic organization of centromeric repeat in Arabidopsis species. Mol Genet Genomics 272: 593-602.

Kawabe A, Hansson B, Hagenblad J, Forrest A, Charlesworth D. 2006. Centromere locations and associated chromosome rearrangements in Arabidopsis lyrata and A. thaliana. Genetics 173: 1613-1619.

Le QH, Wright S, Yu Z, Bureau T. 2000. Transposon diversity in Arabidopsis thaliana. Proc Natl Acad Sci 97: 7376-7381.

Le QH, Melayah D, Bonnivard E, Petit M, Grandbastien MA. 2007. Distribution dynamics of the Tnt 1 retrotransposon in tobacco. Mol Genet Genomics 278: 639-651.

Lippman Z, Gendrel AV, Black M, Vaughn MW, Dedhia N, McCombie WR, Lavine K, Mittal V, May B, Kasschau KD, et al. 2004. Role of transposable elements in heterochromatin and epigenetic control. Nature 430: 471-476.

Ma J, Jackson SA. 2006. Retrotransposon accumulation and satellite amplification mediated by segmental duplication facilitate centromere expansion in rice. Genome Res 16: 251-259.

Madsen LH, Fukai E, Radutoiu S, Yost CK, Sandal N, Schauser L, Stougaard J. 2005. LORE1, an active low-copy-number TY3-gypsy retrotransposon family in the model legume Lotus japonicus. Plant J 44: 372-381.

Mallik HS, Henikoff S. 2002. Conflict begets complexity: The evolution of centromeres. Curr Opin Genet Dev 12: 711-718.

Mirouze M, Reinders J, Bucher E, Nishimura T, Schneeberger K, Ossowski S, Cao J, Weigel D, Paszkowski J, Mathieu O. 2009. Selective epigenetic control of retrotransposition in Arabidopsis. Nature 461: 427-430.

Miura A, Yonebayashi S, Watanabe K, Toyama T, Shimada H, Kakutani T. 2001. Mobilization of transposons by a mutation abolishing full DNA methylation in Arabidopsis. Nature 411: $212-214$.

Nagaki K, Neumann P, Zhang D, Ouyang S, Buell CR, Cheng Z, Jiang J. 2005. Structure, divergence, and distribution of the CRR centromeric retrotransposon family in rice. Mol Biol Evol 22: 845-855.

Okamoto H, Hirochika H. 2000. Efficient insertion mutagenesis of Arabidopsis by tissue culture-induced activation of tobacco retrotransposon Ttol. Plant J 23: 291-304.

Pelissier T, Tutois S, Deragon JM, Tourmente S, Genestier S, Picard G. 1995. Athila, a new retroelement from Arabidopsis thaliana. Plant Mol Biol 29: 441-452.

Peterson-Burch BD, Nettleton D, Voytas DF. 2004. Genomic neighborhoods for Arabidopsis retrotransposons: A role for targeted integration in the distribution of the Metaviridae. Genome Biol 5: R78. doi: 10.1186/gb-2004-5-10-r78.

Rabinowicz PD, Schutz K, Dedhia N, Yordan C, Parnell LD, Stein L, McCombie WR, Martienssen RA. 1999. Differential methylation of genes and retrotransposons facilitates shotgun sequencing of the maize genome. Nat Genet 23: 305-308.

Sandmeyer SB. 1990. Integration specificity of retrotransposons and retroviruses. Annu Rev Genet 24: 491-518.

SanMiguel P, Tikhonov A, Jin YK, Motchoulskaia N, Zakharov D, Melake-Berhan A, Springer PS, Edwards KJ, Lee M, Avramova Z, et al. 1996. Nested retrotransposons in the intergenic regions of the maize genome. Science 274: 765768.

SanMiguel P, Gaut BS, Tikhonov A, Nakajima Y, Bennetzen JL. 1998. The paleontology of intergene retrotransposons of maize. Nat Genet 20: 43-45.

Singer T, Yordan C, Martienssen RA. 2001. Robertson's Mutator transposon in $A$. thaliana are regulated by the chromatinremodeling gene Decrease in DNA methylation (DDM1). Genes Dev 15: 591-602.
Slotkin RK. 2010. The epigenetic control of the Athila family retrotransposons in Arabidopsis. Epigenetics 5: 483-490.

Soppe WI, Jasencakova Z, Houben A, Kakutani T, Meister A, Huang MS, Jacobsen SE, Schubert I, Fransz PF. 2002. DNA methylation controls histone $\mathrm{H} 3$ lysine 9 methylation and heterochromatin assembly in Arabidopsis. EMBO $J$ 21: 6549-6559.

Steimer A, Amedo P, Afsar K, Fransz P, Mittelsten-Scheid O, Paszkowski J. 2000. Endogenous targets of transcriptional gene silencing in Arabidopsis. Plant Cell 12: 1165-1178.

Tsukahara S, Kobayashi A, Kawabe A, Mathieu O, Miura A, Kakutani T. 2009. Bursts of retrotransposition reproduced in Arabidopsis. Nature 461: 423-426.

Vitte C, Bennetzen JL. 2006. Analysis of retrotransposon structural diversity uncovers properties and propensities in angiosperm genome evolution. Proc Natl Acad Sci 103: 1763817643.

Vongs A, Kakutani T, Martienssen RA, Richards EJ. 1993. Arabidopsis thaliana DNA methylation mutants. Science 260: 1926-1928.

Yamazaki M, Tsugawa H, Miyao A, Yano M, Wu J, Yamamoto S, Matsumoto T, Sasaki T, Hirochika H. 2001. Rice retrotransposon Tos17 prefers low-copy-number sequences as integration targets. Mol Genet Genomics 125: 336-344.

Yu W, Han F, Gao Z, Vega JM, Birchler JA. 2007. Construction and behavior of engineered minichromosomes in maize. Proc Natl Acad Sci 104: 8924-8929. 


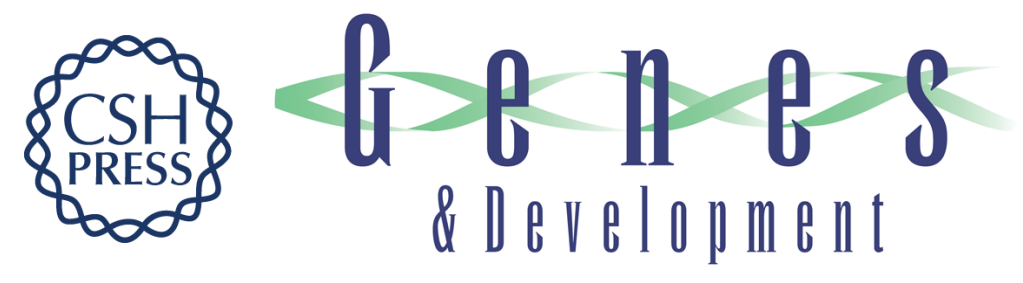

\section{Centromere-targeted de novo integrations of an LTR retrotransposon of Arabidopsis lyrata}

Sayuri Tsukahara, Akira Kawabe, Akie Kobayashi, et al.

Genes Dev. 2012, 26: originally published online March 19, 2012

Access the most recent version at doi:10.1101/gad.183871.111

\section{Supplemental http://genesdev.cshlp.org/content/suppl/2012/03/12/gad.183871.111.DC1 \\ Material}

Related Content Retrotransposon insertion targeting: a mechanism for homogenization of centromere sequences on nonhomologous chromosomes

James A. Birchler and Gernot G. Presting

Genes Dev. April , 2012 26: 638-640

References This article cites 48 articles, 16 of which can be accessed free at: http://genesdev.cshlp.org/content/26/7/705.full.html\#ref-list-1

Articles cited in:

http://genesdev.cshlp.org/content/26/7/705.full.htmI\#related-urls

\section{License}

Email Alerting

Service

Receive free email alerts when new articles cite this article - sign up in the box at the top right corner of the article or click here.

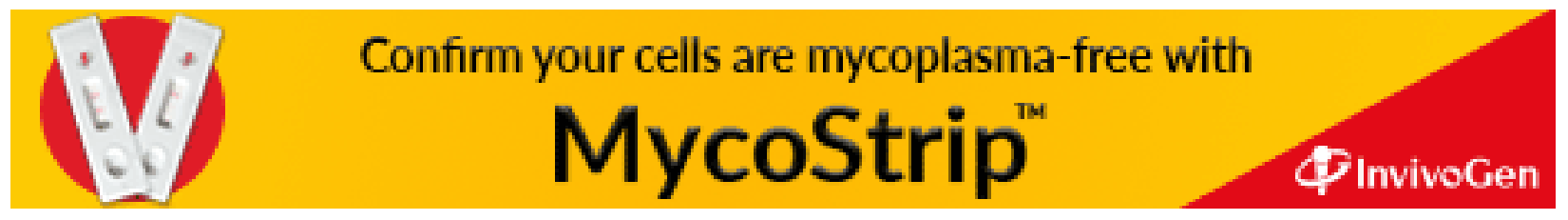

